\title{
Local Existence Result of the Dopant Diffusion in Arbitrary Space Dimensions
}

\author{
R. Bader and W. Merz
}

\begin{abstract}
In this paper we consider the pair diffusion process in more than two spatial dimensions. In this case we are able to prove just a local existence result, since it is not possible to deduce global a priori estimates for the equations as it can be done in the two-dimensional case. The model includes a nonlinear system of reaction-drift-diffusion equations, a nonlinear ordinary differential equation in Banach spaces and an elliptic equation for the electrostatic potential. The local existence result is based on the fixed point theorem of Schauder.
\end{abstract}

Keywords: Dopant diffusion, nonlinear reaction-drift-diffusion equations, ordinary differential equations in Banach spaces

AMS subject classification: 34A34, 34G20, 35J60, 35K45, 35K57

\section{Introduction}

During the doping process impurity atoms of higher or lower chemical valence as silicon are introduced into a silicon layer to influence its electrical properties. Such dopants penetrate under high temperatures, usually around $1000^{\circ} \mathrm{C}$, with the so-called pair diffusion mechanism into the (homogeneous) layer. A precise description of the process can be found in $[1,2,6,12]$ and in the literature cited therein.

Usually, dopant atoms (denoted by $A$ ) occupy substitutional sites in the silicon crystal lattice, loosing (donors, such as Arsenic and Phosphorus) or gaining (acceptors, such as Boron) by this an electron. The dopants move by interacting with native point defects called interstitials (denoted by $I$ ) and vacancies (denoted by $V$ ). Interstitials are silicon atoms which are not placed on a lattice site and move through the crystal unconstrained, and vacancies are empty lattice sites. Both can form mobile pairs with dopant atoms $(A I, A V)$, while the unpaired dopants are immobile. The formation and decay of such

Both authors: Zemtrum Math. der Techn. Univ., Arcisstr. 21, D-80290 München bader@appl-math.tu-muenchen.de and merz@appl-math.tu-muenchen.de

ISSN 0232-2064 / \$2.50 C Heldermann Verlag Berlin 
pairs as well as the recombination of defects cause a movement of the dopants. These interactions can be modelled in terms of chemical reactions. The resulting nonlinear model contains a set of reaction-drift-diffusion equations for the point defects and pairs, reaction equations for the immobile dopants and a Poisson equation for the electrostatic potential, which may not be neglected if the doping concentrations are high, the situation we are concerned with here.

The aim of this paper is to present a local existence result of the nonlinear system in more than two spatial dimensions. However, the derivation of global estimates (with the help of monotonicity properties of energy functionals) is possible only in two spatial dimensions. For this statement, see [4, 7] where global estimates and the asymptotic behaviour of a two-dimensional model, similar to the present one, are investigated. The domain of the system considered there represents a heterogeneous structure, meaning that the coefficients appearing in the equations may have jumps. Whereas strong existence results in two space dimensions can be found in [12 - 15]. The equations there are considered in homogeneous structures (pure silicon wafers for instance) and therefore, the coefficients are smooth functions.

In Sections 2 and 3 we state the mathematical model and summerize the properties of the quantities appearing in the equations. Section 4 is devoted to the nonlinear ordinary differential equation in Banach spaces which describes the evolution of the immobile dopants. We formulate an existence and compactness result, which we will prove with special techniques required for this type of equation. In Section 5 we collect results concerning the Poisson equation. For a detailed analysis of this we refer the reader to the literature. Both, the ordinary differential equation and the Poisson equation are crucial ingradients in Section 6, where we state a local existence and uniqueness result of the complete model. For this we use the fixed point theorem of Schauder. We apply well known embedding theorems in Sobolev spaces and the theory of linear parabolic differential eqations in order to verify the assumptions required in the fixed point theorem. Whereas the uniqueness is an immediate consequence of stability estimates and Gronwall's lemma.

\section{The model}

For $i \in\{I, V, A I, A V, A\}$ we consider the species $X_{i}$ and denote their concentrations by $C_{i}$. We distinguish between mobile and immobile species defining

$$
J=\{I, V, A I, A V\} \quad \text { and } \quad J^{\prime}=\{A\},
$$

respectively. We denote by $\mathbf{C}=\left(C_{I}, C_{V}, C_{A I}, C_{A V}, C_{A}\right)$ the corresponding concentration vector. Each of the $X_{i} \quad\left(i \in J \cup J^{\prime}\right)$ is considered as the union of charged species $X_{i}^{(j)}$, with the charged states $j \in S_{i}$, where each $S_{i} \subset \mathbb{Z}$. 
Thus, if $C_{i}^{(j)}$ denotes the concentration of $X_{i}^{(j)}$, the total concentrations $C_{i}$ are defined as

$$
C_{i}=\sum_{j \in S_{i}} C_{i}^{(j)} \quad\left(i \in J \cup J^{\prime}\right)
$$

The immobile species $X_{i}\left(i \in J^{\prime}\right)$ usually obey one single charged state, which is -1 for acceptors and +1 for donors. In this case we just have $S_{i} \in\{-1,+1\}$.

The charge density of the electrons $n$ and holes $p$ are assumed to obey the Boltzmann statistics, meaning that

$$
n=n_{i} \exp \left(\frac{\psi}{U_{T}}\right) \quad \text { and } \quad p=n_{i} \exp \left(-\frac{\psi}{U_{T}}\right) .
$$

Therein, $n_{i}$ and $U_{T}$ are physical constants.

The chemical potential of the electrons is denoted by $\psi$, and

$$
P_{i}(\psi)=\sum_{j \in S_{i}} K_{i}^{(j)} e^{-j \frac{\psi}{U_{T}}}
$$

are reference concentrations with positive constants $K_{i}^{(j)}$. Set

$$
a_{i}=\frac{C_{i}}{P_{i}(\psi)} \quad\left(i \in J \cup J^{\prime}\right)
$$

which represents the electrochemical activity of the $i$-th component.

We define $Q_{T}=\Omega \times(0, T)$, where $\Omega \subset \mathbb{R}^{n}, 0<T<\infty$ and $3 \leq n \in \mathbb{N}$, with the lateral surface $\Sigma_{T}=\partial \Omega \times(0, T)$. We consider the following equations:

The mobile species for $i \in J$ obey reaction-drift-diffusion equations

$$
\left.\begin{array}{rlrl}
\frac{\partial C_{i}}{\partial t}+\operatorname{div} J_{i} & =R_{i}\left(\left(C_{k}\right)_{k \in J}, C_{A}, \psi\right) & & \text { in } Q_{T} \\
C_{i}(\cdot, 0) & =C_{i}^{0}(\cdot) & & \text { in } \Omega \\
J_{i} \cdot \mathbf{n} & =0 & & \text { on } \Sigma_{T}
\end{array}\right\} .
$$

The immobile dopant concentration $C_{A}$ obeys the reaction equation

$$
\left.\begin{array}{rlrl}
\frac{\partial C_{A}}{\partial t} & =R_{A}\left(\left(C_{k}\right)_{k \in J}, C_{A}, \psi\right) & & \text { in } Q_{T} \\
C_{A}(\cdot, 0) & =C_{A}^{0}(\cdot) & & \text { in } \Omega
\end{array}\right\} .
$$

The Poisson equation for the chemical potential of the electrones reads

$$
\left.\begin{array}{rlrl}
-\frac{\varepsilon}{e} \Delta \psi+2 n_{i} \sinh \left(\frac{\psi}{U_{T}}\right) & =\sum_{i \in J \cup J^{\prime}} Q_{i}(\psi) C_{i} & & \text { in } Q_{T} \\
\nabla \psi \cdot \mathbf{n} & =0 & & \text { on } \Sigma_{T}
\end{array}\right\}
$$


where $\varepsilon$ and $e$ are physical quantities. For $i \in J$ the drift-diffusion term is given by

$$
J_{i}=-D_{i}(\psi)\left\{\nabla C_{i}+Q_{i}(\psi) \nabla\left(\frac{\psi}{U_{T}}\right) C_{i}\right\}
$$

with the diffusivity

$$
D_{i}(\psi)=\sum_{j \in S_{i}} D_{i}^{(j)} K_{i}^{(j)} \frac{e^{-j \frac{\psi}{U_{T}}}}{P_{i}(\psi)}
$$

where $D_{i}^{(j)}$ are positive constants. Whereas,

$$
Q_{i}(\psi)=\sum_{j \in S_{i}} j K_{i}^{(j)} \frac{e^{-j \frac{\psi}{U_{T}}}}{P_{i}(\psi)}
$$

represents the total charge of the $i$-th species for $i \in J \cup J^{\prime}$.

Next, we put the reactions in concrete form. The source terms $R_{i}(\mathbf{C}, \psi)$ result from the reactions occuring during the redistribution of the dopants. All relevant reactions occuring during the (single) dopant diffusion are of second order. We have, due to (2.4),

$$
\begin{aligned}
R_{A, I} & :=K_{A, I}(\psi)\left(a_{A} a_{I}-a_{A I}\right) & R_{A V, I} & :=K_{A V, I}(\psi)\left(a_{A V} a_{I}-a_{A}\right) \\
R_{A, V} & :=K_{A, V}(\psi)\left(a_{A} a_{V}-a_{A V}\right) & R_{A I, V} & :=K_{A I, V}(\psi)\left(a_{A I} a_{V}-a_{A}\right) \\
R_{I, V} & :=K_{I, V}(\psi)\left(a_{I} a_{V}-1\right) & R_{A I, A V} & :=K_{A I, A V}(\psi)\left(a_{A I} a_{A V}-a_{A}^{2}\right)
\end{aligned}
$$

where for certain $i, k \in J \cup J^{\prime}$ the reaction rate coefficients are

$$
K_{i, k}(\psi)=\sum_{j \in S_{i, k}} K_{i, k}^{(j)} e^{-j \frac{\psi}{U_{T}}}
$$

with $K_{i, k}^{(j)}$ positive constants and $S_{i, k} \subset \mathbb{Z}$ special sets of indices. Thus, the source terms $R_{i}(\mathbf{C}, \psi)$ are for $i \in J$ with (2.9) of the form

$$
\begin{aligned}
R_{I}(\mathbf{C}, \psi) & =-R_{A, I}-R_{A V, I}-R_{I, V} \\
R_{V}(\mathbf{C}, \psi) & =-R_{A, V}-R_{A I, V}-R_{I, V} \\
R_{A I}(\mathbf{C}, \psi) & =R_{A, I}-R_{A I, V}-R_{A I, A V} \\
R_{A V}(\mathbf{C}, \psi) & =R_{A, V}-R_{A V, I}-R_{A I, A V}
\end{aligned}
$$

and for $i \in J^{\prime}$ we have

$$
R_{A}(\mathbf{C}, \psi)=-R_{A, I}-R_{A, V}+R_{A I, V}+R_{A V, I}+2 R_{A I, A V} .
$$

For a detailed description and physical meaning of the coefficients mentioned above see, for instance, [2]. Moreover, we set the constants $\varepsilon, e, U_{T}, 2 n_{i}$ equal to one for the analytical investigations. 


\section{Problem (P)}

Now we summerize the basic properties of the coefficients appearing in the equations. The notation of the function spaces corresponds to that in $[8$, 9]. If we consider some function space $Y$, we denote by $Y_{+}$the cone of its non-negative elements. Operations on vectors have to be understood componentwise. Throughout the paper $\Lambda>0$ denotes a generic constant, which we supply with indices if the occasion arises.

As can easily be seen, the coefficients appearing in the equations for $i \in$ $J \cup J^{\prime}$ and $k \in J$ have the following properties:

$$
\begin{aligned}
& D_{k}, Q_{i}, P_{i} \in C^{2}(\mathbb{R}) \\
& 0<\Lambda_{1} \leq D_{k}(\psi) \leq \Lambda_{2} \\
& \left|D_{k}^{(l)}(\psi)\right|,\left|Q_{i}^{(l)}(\psi)\right| \leq \Lambda_{3} \text { with } Q_{i}^{\prime}(\psi)<0 \\
& P_{i}(\psi)=P_{i}(0) \exp \left(-\int_{0}^{\psi} Q_{i}(s) d s\right), P_{i}(0)>0
\end{aligned}
$$

for all $\psi \in \mathbb{R}$ and derivatives $(l=0,1,2)$ of required order two. Furthermore,

$$
0<K_{i, j} \in C^{2}(\mathbb{R})
$$

for certain $i, j \in J \cup J^{\prime}$. Moreover,

$$
P_{i}(\psi), K_{i, j}(\psi) \leq \Lambda_{4} \exp \left(\Lambda_{5}|\psi|\right)
$$

The source terms $(2.10),(2.11)$ obey the growth conditions

$$
\begin{aligned}
& R_{i}(\mathbf{C}, \psi) \leq \lambda_{1}(\psi)\left(\sum_{k \in J \cup J^{\prime}}\left(C_{k}\right)^{2}+1\right)(i \in J) \\
& R_{A}(\mathbf{C}, \psi) \leq-\lambda_{2}(\psi)\left(C_{A}\right)^{2}+\lambda_{3}(\psi)\left(\sum_{k \in J}\left(C_{k}\right)^{2}+1\right),
\end{aligned}
$$

respectively, where $\lambda_{r} \in C(\mathbb{R}) \quad(r=1,2,3)$ with $\lambda_{r}(\psi)>0$ for all $\psi \in \mathbb{R}$ and under the assumption of non-negative concentrations $\mathbf{C}=\left(C_{k}\right)_{k \in J \cup J^{\prime}}$. For $i \in J \cup J^{\prime}$ the source terms satisfy the property

$$
R_{i}(\mathbf{C}, \psi) \geq 0
$$

for all $\psi \in \mathbb{R}$ and $\mathbf{C} \in \mathbb{R}_{+}^{5}$ if $C_{i}=0$. 
Finally, we assume

$$
\left.\begin{array}{l}
\Omega \subset \mathbb{R}^{n} \text { is bounded and } n \geq 3 \\
\partial \Omega \in C^{1,1} \\
C_{i}^{0} \geq 0 \text { in } \Omega \text { for } i \in J \cup J^{\prime} \\
C_{i}^{0} \in W_{p}^{2-\frac{2}{p}}(\Omega) \text { for } i \in J \\
C_{A}^{0} \in C(\bar{\Omega}) \\
p \in(n+2, \infty)
\end{array}\right\}
$$

Since in our case $|J|=4$ and $\left|J^{\prime}\right|=1$ are the numbers of mobile and immobile species, respectively, the formulation of the problem reads as follows:

Definition 1. We denote the system of equations (2.5) - (2.7) by $(\mathbf{P})$ and call the vector $\left(\left(C_{i}\right)_{i \in J}, C_{A}, \psi\right)$ a solution of $(\mathbf{P})$ if

$$
\left(\left(C_{i}\right)_{i \in J}, C_{A}, \psi\right) \in\left[W_{p}^{2,1}\left(Q_{T_{f}}\right)\right]^{4} \times C^{1}\left(\left[0, T_{f}\right] ; C(\bar{\Omega})\right) \times W_{p}^{1}\left(0, T_{f} ; W_{p}^{2}(\Omega)\right)
$$

and satisfies $(\mathrm{P})$ for some $T_{f} \in(0, \infty)$.

\section{Ordinary differential equation}

In this section we consider the ordinary differential equation in Banach spaces (2.6). For given functions $C_{k} \quad(k \in J)$ and $\psi$ with the properties

$$
\begin{aligned}
& C_{k} \geq 0 \\
& C_{k}, \psi \in C([0, T] ; C(\bar{\Omega}))
\end{aligned}
$$

we state an existence result, which we need in the next section.

In accordance with the results and notation used in [11], the reference we apply in the following analysis, we extend problem (2.6) to the whole interval $[0, \infty)$ and write it in the form

$$
\left.\begin{array}{rl}
\dot{u}+\alpha u^{2}+\beta u & =\gamma \quad \text { in }[0, \infty) \\
u(0) & =u_{0}
\end{array}\right\} .
$$

Here the bounded coefficients $\alpha, \beta, \gamma$ are obtained via (2.11), where we have inserted the extented functions $\widetilde{C}_{k}, \widetilde{\psi} \in C([0, \infty) ; C(\bar{\Omega}))$ given by

$$
\widetilde{C}_{k}(t, \cdot)= \begin{cases}C_{k}(t, \cdot) & \text { if } t \in[0, T] \\ C_{k}(T, \cdot) & \text { if } t \in(T, \infty)\end{cases}
$$


(the same with $\psi$ ). With (3.1) and (3.2) we conclude that

$$
\begin{aligned}
& \alpha, \beta, \gamma \in C([0, \infty) ; C(\bar{\Omega})) \\
& \alpha(t, x) \geq c_{0}>0 \text { and } \beta(t, x), \gamma(t, x) \geq 0 \text { in }[0, \infty) \times \bar{\Omega}
\end{aligned}
$$

We have $C_{+}(\bar{\Omega}) \subset C(\bar{\Omega})$ is closed and convex. The map $f$ defined by

$$
\begin{aligned}
& f:[0, \infty) \times C_{+}(\bar{\Omega}) \rightarrow C(\bar{\Omega}) \\
& f(t, u)(x)=\gamma(t, x)-\alpha(t, x) u^{2}(x)-\beta(t, x) u(x)
\end{aligned}
$$

is continuous and maps bounded sets into bounded sets. Moreover, set

$$
m_{ \pm}[v, w]=\lim _{h \rightarrow 0 \pm} \frac{1}{h}\left(\|v+h w\|_{C(\bar{\Omega})}-\|v\|_{C(\bar{\Omega})}\right) .
$$

Then, clearly,

$$
m_{-}[v, w]=-m_{+}[v,-w] .
$$

We apply [11: p. 238/Theorem 5.1] to prove

Lemma 1. Let (4.3) be satisfied. Then problem (4.2) has a unique, nonnegative solution $u \in C^{1}([0, \infty) ; C(\bar{\Omega}))$ which satisfies the estimate

$$
\|u(t)\|_{C(\bar{\Omega})} \leq t\left\|f\left(\cdot, u_{0}\right)\right\|_{C([0, t] ; C(\bar{\Omega}))}+\left\|u_{0}\right\|_{C(\bar{\Omega})}
$$

for all $t \in[0, \infty)$.

Proof. We proceed in Steps I - III.

Step I. We have to ensure that

$$
\liminf _{h \rightarrow 0+} \frac{1}{h} \operatorname{dist}\left(v+h f(t, v), C_{+}(\bar{\Omega})\right)=0
$$

for all $(t, v) \in[0, \infty) \times C_{+}(\bar{\Omega})$. Since $C_{+}(\bar{\Omega})$ is convex, it is sufficient to show that for all $t \in[0, \infty)$ and for all $v \in C_{+}(\bar{\Omega})$ there is a $h_{0}(t, v)>0$ such that, for all $h>0$ with $h \leq h_{0}(t, v)$, the relation $v+h f(t, v) \in C_{+}(\bar{\Omega})$ holds. Using that $\alpha, \beta, \gamma \in C([0, \infty) ; C(\bar{\Omega}))$ with $\gamma(t, x) \geq 0$ and $\alpha(t, x) \geq c_{0}>0$, a short calculation yields

$$
v(x)+h f(t, v)(x) \geq v(x)-h v(x)(\alpha(t, x) v(x)+\beta(t, x)) \geq 0
$$

if $h<\left(\|\beta(t)\|_{C(\bar{\Omega})}+\|\alpha(t)\|_{C(\bar{\Omega})}\|v\|_{C(\bar{\Omega})}\right)^{-1}$.

Step II. We deduce the one-sided estimate

$$
m_{-}[v-w, f(t, v)-f(t, w)] \leq 0
$$


for all $t \in[0, \infty)$ and $v, w \in C_{+}(\bar{\Omega})$. We get

$$
\begin{aligned}
m_{+} & {[v-w,-(f(t, v)-f(t, w))] } \\
= & m_{+}\left[v-w, \alpha(t, \cdot) v^{2}+\beta(t, \cdot) v-\alpha(t, \cdot) w^{2}-\beta(t, \cdot) w\right] \\
= & \lim _{h \rightarrow+0} \frac{1}{h}\left(\left\|(v-w)+h\left(\alpha(t, \cdot) v^{2}+\beta(t, \cdot) v-\alpha(t, \cdot) w^{2}-\beta(t, \cdot) w\right)\right\|_{C(\bar{\Omega})}\right. \\
& \left.\quad-\|v-w\|_{C(\bar{\Omega})}\right) .
\end{aligned}
$$

Let $h>0$. Then

$$
\begin{aligned}
& \left|v(x)-w(x)+h\left(\alpha(t, x) v^{2}(x)+\beta(t, x) v(x)-\alpha(t, x) w^{2}(x)-\beta(t, x) w(x)\right)\right| \\
& \quad=|v(x)-w(x)||1+h \alpha(t, x)(v(x)+w(x))+h \beta(t, x)| \\
& \quad \geq|v(x)-w(x)| .
\end{aligned}
$$

From this and (4.8) we deduce

$$
\|v-w\|_{C(\bar{\Omega})} \leq\left\|(v-w)+h\left(\alpha(t, \cdot) v^{2}+\beta(t, \cdot) v-\alpha(t, \cdot) w^{2}-\beta(t, \cdot) w\right)\right\|_{C(\bar{\Omega})},
$$

i.e. $m_{+}[v-w,-(f(t, v)-f(t, w))] \geq 0$. Thus, from (4.6) we conclude the desired estimate $m_{-}[v-w, f(t, v)-f(t, w)] \leq 0$. So all assumptions of the indicated theorem in [11] are satisfied, saying that for any initial function $u_{0} \in C_{+}(\bar{\Omega})$ there exists a unique solution $u \in C^{1}\left([0, \infty) ; C_{+}(\bar{\Omega})\right)$ of problem (4.2).

Step III. In order to derive estimate (4.7) we set

$$
p(t)=\left\|u(t)-u_{0}\right\|_{C(\bar{\Omega})} \quad(t \in[0, \infty)) .
$$

Then $p$ is left-sided differentiable in $(0, \infty)$ with

$$
\begin{aligned}
p_{-}^{\prime}(t) & =m_{-}\left[u(t)-u_{0}, u^{\prime}(t)\right] \\
& =m_{-}\left[u(t)-u_{0}, f(t, u(t))\right] \\
& \leq m_{-}\left[u(t)-u_{0}, f(t, u(t))-f\left(t, u_{0}\right)\right]+\left\|f\left(t, u_{0}\right)\right\|_{C(\bar{\Omega})} \\
& \leq\left\|f\left(t, u_{0}\right)\right\|_{C(\bar{\Omega})}
\end{aligned}
$$

where the last inequality is a consequence of the previous step. Thus, since $p(0)=0$, we deduce

$$
p(t) \leq \int_{0}^{t}\left\|f\left(s, u_{0}\right)\right\|_{C(\bar{\Omega})} d s \leq t\left\|f\left(\cdot, u_{0}\right)\right\|_{C([0, t] ; C(\bar{\Omega}))} .
$$

In summary we have

$$
\|u(t)\|_{C(\bar{\Omega})} \leq p(t)+\left\|u_{0}\right\|_{C(\bar{\Omega})} \leq t\left\|f\left(\cdot, u_{0}\right)\right\|_{C([0, t] ; C(\bar{\Omega}))}+\left\|u_{0}\right\|_{C(\bar{\Omega})}
$$

which is the desired estimate 
For later use, we state a compactness result concerning problem (4.2). Let $n+2<p<\infty$ and $\alpha, \beta, \gamma \in C\left([0, T] ; C_{+}(\bar{\Omega})\right) \cap L^{1}\left(0, T ; W_{p}^{1}(\Omega)\right)$. Then we define the operator

$$
\left.\begin{array}{l}
L:\left[C\left([0, T] ; C_{+}(\bar{\Omega})\right) \cap L^{1}\left(0, T ; W_{p}^{1}(\Omega)\right)\right]^{3} \rightarrow C\left([0, T] ; C_{+}(\bar{\Omega})\right) \\
L(\alpha, \beta, \gamma)=u
\end{array}\right\}
$$

where $u$ is a solution of problem (4.2).

We use Ascoli's theorem (see [11]) to state the following

Lemma 2. Let (4.3) be satisfied. Then the mapping stated in (4.9) is compact.

Proof. Let

$$
\left\{\left(\alpha_{n}, \beta_{n}, \gamma_{n}\right)\right\}_{n \in \mathbb{N}} \subset\left[C\left([0, T] ; C_{+}(\bar{\Omega})\right) \cap L^{1}\left(0, T ; W_{p}^{1}(\Omega)\right)\right]^{3}
$$

be a sequence satisfying

$$
\left\|\left(\alpha_{n}, \beta_{n}, \gamma_{n}\right)\right\|_{\left[C\left([0, T] ; C_{+}(\bar{\Omega})\right)\right]^{3}}+\left\|\left(\alpha_{n}, \beta_{n}, \gamma_{n}\right)\right\|_{\left[L^{1}\left(0, T ; W_{p}^{1}(\Omega)\right)\right]^{3}} \leq \bar{\Lambda}
$$

with a constant $\bar{\Lambda}>0$. We consider $\left\{u_{n}\right\}_{n \in \mathbb{N}} \subset C\left([0, T] ; C_{+}(\bar{\Omega})\right)$ defined by $u_{n}=L\left(\alpha_{n}, \beta_{n}, \gamma_{n}\right)$ and again proceed in several steps.

Step I. We have to show that $\left\{u_{n}\right\}_{n \in \mathbb{N}}$ is equicontinuous. Let

$$
f_{n}(t, v)=\gamma_{n}(t, \cdot)-\alpha_{n}(t, \cdot) v^{2}-\beta_{n}(t, \cdot) v
$$

For $s<t$ we have the estimate

$$
\left\|u_{n}(t)-u_{n}(s)\right\|_{C(\bar{\Omega})} \leq \int_{s}^{t}\left\|f_{n}\left(\tau, u_{n}(\tau)\right)\right\|_{C(\bar{\Omega})} \leq(t-s) \tilde{\Lambda}
$$

where the constant $\tilde{\Lambda}>0$ is independent of $n$. This proves the equicontinuity.

II. Finally, we have to verify that for any $t \in[0, T]$ the set $\left\{u_{n}(t)\right\}_{n \in \mathbb{N}} \subset$ $C(\bar{\Omega})$ is relatively compact. We apply the theorem of Ascoli-Arcelá.

1. From (4.7) we get the estimate

$$
\left|u_{n}(t)(x)\right| \leq\left\|u_{n}(t)\right\|_{C(\bar{\Omega})} \leq T \tilde{\Lambda}+\left\|u_{0}\right\|_{C(\bar{\Omega})}=: \hat{\Lambda}
$$

for all $x \in \bar{\Omega}$, which is independent on $n \in \mathbb{N}$. 
2.) It remains to prove the equicontinuity in $\bar{\Omega}$. Let $x \neq y \in \bar{\Omega}$. A short calculation and the application of Gronwall's lemma yield

$$
\begin{aligned}
& \left|u_{n}(t)(x)-u_{n}(t)(y)\right| \\
& \quad \leq \exp \left(\Lambda_{1} T\right) \Lambda_{2}\left(\left|u_{0}(x)-u_{0}(y)\right|+\int_{0}^{T}\left|\alpha_{n}(s, x)-\alpha_{n}(s, y)\right| d s\right. \\
& \left.\quad+\int_{0}^{T}\left|\beta_{n}(s, x)-\beta_{n}(s, y)\right| d s+\int_{0}^{T}\left|\gamma_{n}(s, x)-\gamma_{n}(s, y)\right| d s\right)
\end{aligned}
$$

for all $t \in[0, T]$ and some constants $\Lambda_{1}$ and $\Lambda_{2}$, which are composed of the quantities $\bar{\Lambda}$ and $\hat{\Lambda}$ introduced in the present derivation. Since $\alpha_{n}, \beta_{n}, \gamma_{n} \in$ $L^{1}\left(0, T ; W_{p}^{1}(\Omega)\right)$, it results from embedding theorems (see [8]) that $\alpha_{n}, \beta_{n}, \gamma_{n} \in$ $C^{\lambda}(\bar{\Omega})$ with $0<\lambda \leq 1-\frac{n}{p}$. Thus we get

$$
\begin{aligned}
\int_{0}^{T} \frac{\left|\alpha_{n}(s, x)-\alpha_{n}(s, y)\right|}{\|x-y\|^{\lambda}} d s\|x-y\|^{\lambda} & \leq \int_{0}^{T}\left\|\alpha_{n}(s)\right\|_{C^{\lambda}(\bar{\Omega})} d s\|x-y\|^{\lambda} \\
& \leq T \Lambda\|x-y\|^{\lambda}
\end{aligned}
$$

and similarily with $\beta_{n}$ and $\gamma_{n}$. We conclude with

$$
\left|u_{n}(t)(x)-u_{n}(t)(y)\right| \leq \Lambda\left(\left|u_{0}(x)-u_{0}(y)\right|+\|x-y\|^{\lambda}\right)
$$

which yields the desired equicontinuity and completes the proof of compactness

\section{Poisson equation}

Next, we collect results concerning the elliptic equation (2.7). We sketch the results and refer the reader for a detailed analysis to $[12,15]$. Therein, the existence results are restricted to two spatial dimensions as a consequence of an $L^{\infty}$-estimate for the chemical potential of the electrones and the embedding theorem of Trudinger [16]. We do not need these ingradients in the subsequent sections. Thus, the following results regarding the Poisson equation are valid in any space dimension.

We use the fixed point theorem of Leray-Schauder (see [3]) to prove the following

Lemma 3. Let $n<p<\infty$ and $\mathbf{C} \in\left[L_{+}^{p}(\Omega)\right]^{5}$. Then there exists a unique solution $\psi \in W_{p}^{2}(\Omega)$ of problem (2.7). Moreover, there exists a constant $\Lambda_{p}>0$ such that

$$
\|\psi-\tilde{\psi}\|_{W_{p}^{2}(\Omega)} \leq \Lambda_{p} \sum_{i \in J \cup J^{\prime}}\left\|C_{i}-\tilde{C}_{i}\right\|_{L^{p}(\Omega)}
$$


for $\mathbf{C}, \tilde{\mathbf{C}} \in\left[L_{+}^{p}(\Omega)\right]^{5}$ and the corresponding $\psi, \tilde{\psi}$ satisfying the Poisson equation.

Proof. We define the mapping

$$
Z: W_{p}^{1}(\Omega) \times[0,1] \rightarrow W_{p}^{1}(\Omega), \quad Z(\phi, \tau)=\psi
$$

where $\psi$ is the solution of the linear problem

$$
\left.\begin{array}{rlrl}
-\Delta \psi+\lambda \psi & =\tau\left(\lambda \phi-\sinh \phi+\sum_{i \in J \cup J^{\prime}} Q_{i}(\phi) C_{i}\right):=\tau g & & \text { in } \Omega \\
\nabla \psi \cdot \mathbf{n} & =0 & & \text { on } \partial \Omega
\end{array}\right\}
$$

with an appropriate constant $\lambda>0$. We proceed in Steps I - V.

Step I. The mapping $Z$ is well defined, since Sobolev's lemma yields $\phi \in C(\bar{\Omega})$, and so does $\sinh (\phi)$. This combined with (3.1) results in $g \in L^{p}(\Omega)$. Thus, from the elliptic theory (see $[5,9]$ ) we conclude the existence of a unique solution $\psi \in W_{p}^{2}(\Omega)$ of problem $(5.2)$.

II. $Z(\phi, 0)=0$ for all $\phi \in W_{p}^{1}(\Omega)$.

III. From the compact embedding of $W_{p}^{2}(\Omega)$ into $W_{p}^{1}(\Omega)$ we get the compactness of the mapping $Z$.

IV. Obviously, for $\phi$ from bounded sets in $W_{p}^{1}(\Omega)$ the mapping $Z(\phi, \cdot)$ is uniformly continuous. We show the continuity in the first argument. Let $\tau=1$ and set $\bar{\psi}=\psi_{1}-\psi_{2}$ as well as $\bar{\phi}=\phi_{1}-\phi_{2}$. Moreover, we deduce from the mean value theorem

$$
\sinh \left(\phi_{1}\right)-\sinh \left(\phi_{2}\right)=\int_{0}^{1} \sinh (\tilde{\phi}(s)) d s \bar{\phi}
$$

(similarily with $\left.Q_{i}\right)$ where $\tilde{\phi} \in C(\bar{\Omega}), \tilde{\phi}(s)=s \phi_{1}(s)+(1-s) \phi_{2}(s) \quad(s \in[0,1])$. We get

$$
\left.\begin{array}{rlr}
-\Delta \bar{\psi}+\lambda \bar{\psi} & =\lambda \bar{\phi}-\int_{0}^{1} \cosh (\tilde{\phi}) d s \bar{\phi}+\sum_{i} \int_{0}^{1} Q_{i}^{\prime}(\tilde{\phi}) d s \bar{\phi} C_{i} & \text { in } \Omega \\
\nabla \bar{\psi} \cdot \mathbf{n} & =0 & \text { on } \partial \Omega
\end{array}\right\} .
$$

From the linear elliptic theory and Sobolev's embedding theorem we conclude that there exists a constant $\Lambda>0$ such that

$$
\|\bar{\psi}\|_{W_{p}^{2}(\Omega)} \leq \Lambda\left(\|\bar{\phi}\|_{L_{p}(\Omega)}+\sum_{i \in J \cup J^{\prime}}\left\|C_{i}\right\|_{L_{p}(\Omega)}\|\bar{\phi}\|_{C(\bar{\Omega})}\right) \leq \Lambda\|\bar{\phi}\|_{W_{p}^{1}(\Omega)}
$$


from which the continuity follows.

Step V. In order to derive the $\tau$-independent a priori estimates in $W_{p}^{2}(\Omega)$ of the fixed points we start to test the corresponding equation with $\psi|\psi|^{p-2}$ to deduce for $\tau \in\left[0, \frac{1}{2}\right]$

$$
\begin{gathered}
\int_{\Omega}-\Delta \psi \psi|\psi|^{p-2} d x+(1-\tau) \lambda \int_{\Omega}|\psi|^{p} d x+\tau \int_{\Omega} \sinh (\psi) \psi|\psi|^{p-2} d x \\
=\tau \int_{\Omega} \sum_{i \in J \cup J^{\prime}} Q_{i}(\psi) C_{i} \psi|\psi|^{p-2} d x .
\end{gathered}
$$

Obviously, the first and the third integral are non-negative. So we get with Young's inequality

$$
\frac{1}{2} \lambda \int_{\Omega}|\psi|^{p} d x \leq \Lambda_{\delta} \sum_{i \in J \cup J^{\prime}} \int_{\Omega}\left|C_{i}\right|^{p} d x+f(\delta) \int_{\Omega}|\psi|^{p} d x
$$

where $f(\delta) \rightarrow 0$ as $\delta \rightarrow 0$. Thus, taking $\delta>0$ small, there exists a constant $\Lambda_{p}>0$ such that

$$
\|\psi\|_{L^{p}(\Omega)} \leq \Lambda_{p} \sum_{i \in J \cup J^{\prime}}\left\|C_{i}\right\|_{L^{p}(\Omega)} .
$$

Next, we test with $\sinh (\psi)|\sinh (\psi)|^{p-2}$ to get for $\tau \in\left[\frac{1}{2}, 1\right]$

$$
\begin{gathered}
\int_{\Omega}-\Delta \psi \sinh (\psi)|\sinh (\psi)|^{p-2} d x \\
+(1-\tau) \lambda \int_{\Omega} \psi \sinh (\psi)|\sinh (\psi)|^{p-2} d x \\
+\tau \int_{\Omega}|\sinh (\psi)|^{p} d x=\tau \int_{\Omega} \sum_{i \in J \cup J^{\prime}} Q(\psi) C_{i} \sinh (\psi)|\sinh (\psi)|^{p-2} d x .
\end{gathered}
$$

The first (see, e.g., [15]) and the second integral on the left-hand side are nonnegative. Again, Young's inequality yields the existence of a constant $\Lambda_{p}>0$ such that

$$
\|\sinh (\psi)\|_{L^{p}(\Omega)} \leq \Lambda_{p} \sum_{i \in J \cup J^{\prime}}\left\|C_{i}\right\|_{L^{p}(\Omega)} .
$$

Since $|\psi| \leq|\sinh (\psi)|$ we get from (5.3) and (5.4) that

$$
\|\psi\|_{L^{p}(\Omega)} \leq \Lambda_{p} \sum_{i \in J \cup J^{\prime}}\left\|C_{i}\right\|_{L^{p}(\Omega)}
$$

which is independent of $\tau$. 
Finally, we take $-\Delta \psi|\Delta \psi|^{p-2}$ as a test function, use (5.5) and the inequality of Young, saying that there exists a constant $\Lambda_{p}>0$ such that

$$
\|\Delta \psi\|_{L^{p}(\Omega)} \leq \Lambda_{p} \sum_{i \in J \cup J^{\prime}}\left\|C_{i}\right\|_{L^{p}(\Omega)} .
$$

With (5.5) and (5.6) the elliptic theory comes out with

$$
\|\psi\|_{W_{p}^{2}(\Omega)} \leq \Lambda_{p} \sum_{i \in J \cup J^{\prime}}\left\|C_{i}\right\|_{L^{p}(\Omega)}
$$

which is the desired estimate.

Using the non-negativity of the concentration vector $\left(C_{i}\right)_{i \in J \cup J^{\prime}}$ it is easy to prove stability estimate (5.1) from which the uniqueness of the solution of the Poisson equation follows

We include the time regularity of $\psi$. So, if $C_{i} \in C\left([0, T] ; L^{p}(\Omega)\right)$ for $i \in J \cup J^{\prime}$, then we immediately get

$$
\psi \in C\left([0, T] ; W_{p}^{2}(\Omega)\right) .
$$

Moreover, there exists a constant $\Lambda_{p}>0$ such that

$$
\|\psi\|_{C\left([0, T] ; W_{p}^{2}(\Omega)\right)} \leq \Lambda_{p} \sum_{i \in J \cup J^{\prime}}\left\|C_{i}\right\|_{C\left([0, T] ; L^{p}(\Omega)\right)} .
$$

If in addition $C_{i} \in W_{p}^{1}\left(0, T ; L^{p}(\Omega)\right) \cap C([0, T] ; C(\bar{\Omega}))$ for $i \in J \cup J^{\prime}$, we are able to show that

$$
\psi \in W_{p}^{1}\left(0, T ; W_{p}^{2}(\Omega)\right)
$$

and that there exists another constant $\Lambda_{p}>0$ satisfying

$$
\|\psi\|_{W_{p}^{1}\left(0, T ; W_{p}^{2}(\Omega)\right)} \leq \Lambda_{p} \sum_{i \in J \cup J^{\prime}}\left\|C_{i}\right\|_{W_{p}^{1}\left(0, T ; L^{p}(\Omega)\right)} .
$$

Thus, we have summarized all results concerning $\psi$, which we need for further investigations. 


\section{Existence and uniqueness}

Using the fixed point theorem of Schauder, we prove the existence of a strong solution of Problem (P) according to Definition 1. We are able to formulate the following main result:

Theorem 1. Under assumptions (3.1) - (3.7) there exists an instant of time $T_{f}>0$ such that the system of equations $(2.5)-(2.7)$ has a unique solution $\mathbf{C}$. This solution satisfies $\mathbf{C} \geq 0$.

The proof of this theorem consists of several steps, which we present in the next subsections. We start with a modification of our problem.

Definition 2. If we replace in problem (P) the source terms by $R_{i}\left(\left(C_{k}^{+}\right)_{k \in J}, C_{A}\right.$, $\psi$ ) and the right-hand side in Poisson equation $(2.7)$ by $\sum_{i \in J} Q_{i}(\psi) C_{i}^{+}+$ $Q_{A}(\psi) C_{A}$ where

$$
C_{i}^{+}= \begin{cases}C_{i} & \text { if } C_{i} \geq 0 \\ 0 & \text { if } C_{i}<0\end{cases}
$$

we denote the modified system by $\left(\mathrm{P}^{+}\right)$.

In the next subsection we will show that for any solution of problem $\left(\mathrm{P}^{+}\right)$ the concentrations are non-negative. Then we will prove the existence of a strong solution of problem $\left(\mathrm{P}^{+}\right)$with the help of Schauder's fixed point theorem in Sobolev spaces and use regularity results to get the desired smoothness. This (non-negative) solution obviously solves problem $(\mathrm{P})$, too. Finally, we have to show that there exists no other solution of problem $(\mathrm{P})$, which concludes the proof of Theorem 1 .

6.1 Problem $\left(\mathbf{P}^{+}\right)$. First we prove the non-negativity of the concentrations of the mobile species.

Lemma 4. Let

$$
\left(\left(C_{i}\right)_{i \in J}, C_{A}, \psi\right) \in\left[W_{p}^{2,1}\left(Q_{T_{f}}\right)\right]^{4} \times C^{1}\left(\left[0, T_{f}\right] ; C(\bar{\Omega})\right) \times W_{p}^{1}\left(0, T_{f} ; W_{p}^{2}(\Omega)\right)
$$

be a solution of problem $\left(\mathrm{P}^{+}\right)$. Then $C_{i} \geq 0$ for $i \in J$.

Proof. For $i \in J$ we test the equation

$$
\frac{\partial C_{i}}{\partial t}+\operatorname{div} J_{i}=R_{i}\left(\left(C_{k}^{+}\right)_{k \in J}, C_{A}, \psi\right)
$$


with $C_{i}^{-}=C_{i}^{+}-C_{i}$ where $J_{i}$ is defined in (2.8). We get with appropriate constants the estimate

$$
\begin{gathered}
\int_{\Omega}\left(C_{i}^{-}(t)\right)^{2} d x+\int_{0}^{t} \int_{\Omega}\left(\Lambda_{1}\left(\nabla C_{i}^{-}\right)^{2}+R_{i}\left(\left(C_{k}^{+}\right)_{k \in J}, C_{A}, \psi\right) C_{i}^{-}\right) d x d s \\
\leq \Lambda\left(\frac{\varepsilon}{2} \int_{0}^{t} \int_{\Omega}\left(\nabla C_{i}^{-}\right)^{2} d x d s+\Lambda_{\varepsilon} \int_{0}^{t} \int_{\Omega}(\nabla \psi)^{2}\left(C_{i}^{-}\right)^{2} d x d s\right) \\
\leq \Lambda\left(\frac{\varepsilon}{2} \int_{0}^{t} \int_{\Omega}\left(\nabla C_{i}^{-}\right)^{2} d x d s+\Lambda_{\varepsilon} \int_{0}^{t}\|\nabla \psi\|_{C(\bar{\Omega})}^{2}\left\|C_{i}^{-}\right\|_{L^{2}(\Omega)}^{2} d s\right)
\end{gathered}
$$

where we used Young's inequality and properties (3.1) - (3.2). Since $C_{i}^{+} C_{i}^{-}=$ 0 we are able to apply property (3.6) to omit the reaction rates. We choose $\varepsilon>0$ such that $\frac{\Lambda \varepsilon}{2}=\Lambda_{1}$. Then we get

$$
\left\|C_{i}^{-}(t)\right\|_{L^{2}(\Omega)}^{2} \leq \Lambda \int_{0}^{t}\|\nabla \psi\|_{C(\bar{\Omega})}^{2}\left\|C_{i}^{-}\right\|_{L^{2}(\Omega)}^{2} d s .
$$

We have $\nabla \psi \in L^{2}(0, T ; C(\bar{\Omega}))$ and $C_{i}^{-} \in C\left([0, T] ; L^{2}(\Omega)\right)$. So we can use Gronwall's lemma, saying that $\left\|C_{i}^{-}(t)\right\|_{L^{2}(\Omega)}^{2}=0$ for all $t \in[0, T]$

6.2 Fixed point iteration for problem $\left(\mathbf{P}^{+}\right)$. Now we prove the existence of a local solution of problem $\left(\mathrm{P}^{+}\right)$in Sobolev spaces by means of the fixed point theorem of Schauder.

We define the constants

$$
\begin{aligned}
& k_{1}=K_{0} \Lambda_{0} \\
& k_{2}=k\left(k_{1}+\Lambda_{0}\right) .
\end{aligned}
$$

with

$$
\begin{aligned}
\frac{\Lambda_{0}}{2} & =\sum_{i \in J}\left\|C_{i}^{0}\right\|_{W_{p}^{2-\frac{2}{p}}(\Omega)}+\left\|C_{A}^{0}\right\|_{C(\bar{\Omega})}+1 \\
K_{0} & =\sum_{i \in J} K_{i}
\end{aligned}
$$

where the constants $K_{i}, k>0$ depend on known quantities only and will be specified below. Further, we define the set

$$
X_{T}=\left\{\begin{array}{l|l}
(\mathcal{C}, \phi) \in\left[W_{p}^{2,1}\left(Q_{T}\right)\right]^{4} \times C\left([0, T] ; W_{p}^{1}(\Omega)\right) & \begin{array}{c}
\|\mathcal{C}\|_{\left[W_{p}^{2,1}\left(Q_{T}\right)\right]^{4}} \leq k_{1} \\
\|\phi\|_{C\left([0, T] ; W_{p}^{1}(\Omega)\right)} \leq k_{2}
\end{array}
\end{array}\right\}
$$


for some $T \in(0, \infty)$ and consider the vector-valued mapping

$$
\left.\begin{array}{l}
Z: X_{T} \rightarrow\left[W_{p}^{2,1}\left(Q_{T}\right)\right]^{4} \times C\left([0, T] ; W_{p}^{1}(\Omega)\right) \\
Z\left(\left(\mathcal{C}_{k}\right)_{k \in J}, \phi\right)=\left(\left(C_{k}\right)_{k \in J}, \psi\right)
\end{array}\right\}
$$

where $C_{A}$ is the non-negative solution of the ordinary differential equation problem in Banach spaces

$$
\left.\begin{array}{rlrl}
\frac{\partial C_{A}}{\partial t} & =R_{A}\left(\left(\mathcal{C}_{k}^{+}\right)_{k \in J}, C_{A}, \phi\right) & & \text { in } Q_{T} \\
C_{A}(\cdot, 0) & =C_{A}^{0} & & \text { in } \Omega
\end{array}\right\}
$$

the chemical potential $\psi$ is the solution of the problem

$$
\left.\begin{array}{rlrl}
-\Delta \psi+\sinh \psi & =\sum_{i \in J} Q_{i}(\psi) \mathcal{C}_{i}^{+}+Q_{A}(\psi) C_{A} & & \text { in } Q_{T} \\
\nabla \psi \cdot \mathbf{n} & =0 & & \text { on } \Gamma_{T}
\end{array}\right\}
$$

and for $i \in J$ the concentrations $C_{i}$ solve the problem

$$
\left.\begin{array}{rlrl}
\frac{\partial C_{i}}{\partial t}-\operatorname{div}\left\{D_{i}(\psi)\left[\nabla C_{i}+Q_{i}(\psi) \nabla \psi C_{i}\right]\right\} & =R_{i}\left(\left(\mathcal{C}_{k}^{+}\right)_{k \in J}, C_{A}, \psi\right) & & \text { in } Q_{T} \\
\nabla C_{i} \cdot \mathbf{n} & =0 & & \text { on } \Gamma_{T} \\
C_{i}(\cdot, 0) & =C_{i}^{0} & & \text { in } \Omega
\end{array}\right\}
$$

Now we check the properties of the mapping required in the fixed point theorem in the following steps Ia/b - III:

Step Ia. The mapping $Z$ is well defined, since system (6.5) - (6.7) has a unique solution

$$
\left(C_{A}, \psi,\left(C_{i}\right)_{i \in J}\right) \in C^{1}([0, T] ; C(\bar{\Omega})) \times W_{p}^{1}\left(0, T ; W_{p}^{2}(\Omega)\right) \times\left[W_{p}^{2,1}\left(Q_{T}\right)\right]^{4}
$$

Indeed, in order to see the solvability of system (6.5) - (6.7) we first note that for $i \in J$ each $\mathcal{C}_{i} \in W_{p}^{2,1}\left(Q_{T}\right)$, due to embedding theorems, also belongs to the space $C([0, T] ; C(\bar{\Omega}))$ and so do the cuts. The function $\phi \in C\left([0, T] ; W_{p}^{1}(\Omega)\right)$ is also continuous in both variables. Having this in mind we can say that for given $\left(\left(\mathcal{C}_{i}\right)_{k \in J}, \phi\right) \in[C([0, T] ; C(\bar{\Omega}))]^{5}$ according to Lemma 1 the nonlinear equation (6.5) has a unique solution

$$
C_{A} \in C^{1}([0, T] ; C(\bar{\Omega}))
$$


which satisfies $C_{A} \geq 0$. In addition, the concentrations $C_{A}$ and $\mathcal{C}_{i} \quad(i \in J)$ in the right-hand side of $(6.6)$ belong to the space $W_{p}^{1}\left(0, T ; L^{p}(\Omega)\right)$. This yields due to (5.10) that

$$
\psi \in W_{p}^{1}\left(0, T ; W_{p}^{2}(\Omega)\right) .
$$

From (6.9), (6.10) and embedding theorems it follows that the coefficients as well as the right-hand side of (6.7) are continuous, and thus they belong to the space $L^{p}\left(Q_{T}\right)$ for any $p \geq 1$, especially for $p \in(n+2, \infty)$. So, with (3.7) the parabolic theory (see [8]) yields

$$
\left(C_{i}\right)_{i \in J} \in\left[W_{p}^{2,1}\left(Q_{T}\right)\right]^{4}
$$

and (6.8) follows.

Step Ib. For later use, we state an estimate. We get by testing (6.5) with $\frac{\partial}{\partial t}\left(C_{A}^{1}-C_{A}^{2}\right)\left|\frac{\partial}{\partial t}\left(C_{A}^{1}-C_{A}^{2}\right)\right|^{p-2}$, combined with the linear theory of ordinary differential equations in Banach spaces, and from the linear elliptic theory applied to (6.6) that there exists a constant $\Lambda>0$ such that the stability estimate

$$
\begin{aligned}
\| C_{A}^{1} & -C_{A}^{2}\left\|_{W_{p}^{1}\left(0, T ; L^{p}(\Omega)\right)}+\right\| \psi_{1}-\psi_{2} \|_{L^{p}\left(0, T ; W_{p}^{2}(\Omega)\right)} \\
& \leq \Lambda\left(\sum_{i \in J}\left\|\mathcal{C}_{i}^{1+}-\mathcal{C}_{i}^{2+}\right\|_{L^{p}\left(0, T ; L^{p}(\Omega)\right)}+\left\|\phi_{1}-\phi_{2}\right\|_{L^{p}\left(0, T ; L^{p}(\Omega)\right)}\right)
\end{aligned}
$$

holds for all $\phi_{1}, \phi_{2}, \mathcal{C}_{i}^{1+}, \mathcal{C}_{i}^{2+} \in L^{p}\left(0, T ; L^{p}(\Omega)\right)$ with the corresponding solutions $C_{A}^{1}, C_{A}^{2}$ of problem (6.5) and $\psi_{1}, \psi_{2}$ of problem (6.6).

Step II. We show that there exists an instant of time $T_{f} \in(0, \infty)$ such that $Z\left(X_{T_{f}}\right) \subseteq X_{T_{f}}$. Let $T_{0} \in(0, \infty)$ be fixed. In order to state the constant $k>0$ used in (6.1) we estimate the chemical potential given by equation (6.6). According to (4.7), (5.9) and (6.3) there exists (cf. (5.9)) a constant $k>0$ such that

$$
\begin{aligned}
\|\psi\|_{C\left([0, T] ; W_{p}^{2}(\Omega)\right)} & \leq k\left(\sum_{i \in J}\left\|\mathcal{C}_{i}^{+}\right\|_{C\left([0, T] ; L^{p}(\Omega)\right)}+\left\|C_{A}\right\|_{C\left([0, T] ; L^{p}(\Omega)\right)}\right) \\
& \leq k\left(k_{1}+\frac{\Lambda_{0}}{2}+T \Lambda_{\Omega}\left\|R_{A}\left(\left(\mathcal{C}_{k}^{+}\right)_{k \in J}, C_{A}^{0}, \phi\right)\right\|_{C\left(\bar{Q}_{T}\right)}\right)
\end{aligned}
$$

where $\Lambda_{\Omega}>0$ and $R_{A}\left(\left(\mathcal{C}_{k}^{+}\right)_{k \in J}, C_{A}^{0}, \phi\right)$ depends on known quantities, especially on $k_{1}, k_{2}>0$ stated in (6.3). We choose $T_{1} \in\left(0, T_{0}\right]$ such that

$$
T_{1} \Lambda_{\Omega}\left\|R_{A}\left(\left(\mathcal{C}_{k}^{+}\right)_{k \in J}, C_{A}^{0}, \phi\right)\right\|_{C\left(\bar{Q}_{t}\right)} \leq \frac{\Lambda_{0}}{2}
$$


and conclude

$$
\|\psi\|_{C\left(\left[0, T_{1}\right] ; W_{p}^{1}(\Omega)\right)} \leq\|\psi\|_{C\left(\left[0, T_{1}\right] ; W_{p}^{2}(\Omega)\right)} \leq k_{2}
$$

Moreover, the local solution $\psi \in W_{p}^{1}\left(0, T_{1} ; W_{p}^{2}(\Omega)\right)$ satisfies estimate $(5.11)$ with $\mathcal{C}_{i}^{+}$instead of $C_{i}$ for $i \in J$ therein.

In order to state $K_{i}>0$ defined in (6.2), let us write the differential equation in problem (6.7) for $i \in J$ in the form

$$
\frac{\partial C_{i}}{\partial t}-D_{i}(\psi) \Delta C_{i}=F_{i}
$$

where

$$
F_{i}=R_{i}\left(\left(\mathcal{C}_{k}^{+}\right)_{k \in J}, C_{A}, \psi\right)+\operatorname{div}\left\{D_{i}(\psi) Q_{i}(\psi) \nabla \psi C_{i}\right\}+D^{\prime}(\psi) \nabla \psi \cdot \nabla C_{i}
$$

We set $K_{i}=K_{i}\left(T_{0}\right)$. Then the parabolic theory yields the estimate

$$
\left\|C_{i}\right\|_{W_{p}^{2,1}\left(Q_{T}\right)} \leq \frac{K_{i}}{2}\left(\left\|C_{i}^{0}\right\|_{W_{p}^{2-\frac{2}{p}}(\Omega)}+\left\|F_{i}\right\|_{L^{p}\left(0, T ; L^{p}(\Omega)\right)}\right)
$$

which is true for all $T \in\left(0, T_{0}\right]$ and where the $K_{i}>0$ remain bounded for any finite $T_{0}>0$ (see [8]). With (6.16) we get the estimates

$$
\begin{aligned}
\left\|C_{i}\right\|_{W_{p}^{2,1}\left(Q_{T}\right)} \leq \frac{K_{0}}{2}\left(\frac{\Lambda_{0}}{2}\right. & +\left\|R_{i}\left(\left(\mathcal{C}_{k}^{+}\right)_{k \in J}, C_{A}, \psi\right)\right\|_{L^{p}\left(0, T ; L^{p}(\Omega)\right)} \\
& +\left\|\operatorname{div}\left\{D_{i}(\psi) Q_{i}(\psi) \nabla \psi C_{i}\right\}\right\|_{L^{p}\left(0, T ; L^{p}(\Omega)\right)} \\
& \left.+\left\|D^{\prime}(\psi) \nabla \psi \cdot \nabla C_{i}\right\|_{L^{p}\left(0, T ; L^{p}(\Omega)\right)}\right)
\end{aligned}
$$

for $i \in J$ and where the constants $K_{0}, \Lambda_{0}$ are defined in (6.2).

We estimate the first $L^{p}$-norm in (6.17). There exists a constant $\Lambda_{1}>0$, just depending on known quantities (especially on $k_{1}, k_{2}>0$ ) such that

$$
\begin{aligned}
& \left\|R_{i}\left(\left(\mathcal{C}_{k}^{+}\right)_{k \in J}, C_{A}, \psi\right)\right\|_{L^{p}\left(0, T ; L^{p}(\Omega)\right)} \\
& \quad \leq T^{\frac{1}{p}} \Lambda_{\Omega}\left\|\lambda_{i}(\psi)\right\|_{C([0, T] ; C(\bar{\Omega}))} \\
& \quad \times\left(\sum_{k \in J}\left\|\left(\mathcal{C}_{k}^{+}\right)^{2}\right\|_{C([0, T] ; C(\bar{\Omega}))}+\left\|\left(C_{A}\right)^{2}\right\|_{C([0, T] ; C(\bar{\Omega}))}+1\right) \\
& \quad \leq T^{\frac{1}{p}} \Lambda_{1}
\end{aligned}
$$


where $\lambda_{i} \in C(\mathbb{R}) \quad(i \in J)$. We consider the second $L^{p}$-norm in (6.17) which is

$$
\begin{aligned}
\operatorname{div}\left\{D_{i}(\psi) Q_{i}(\psi) \nabla \psi C_{i}\right\}= & \left(D_{i}^{\prime}(\psi) Q_{i}(\psi)+D_{i}(\psi) Q_{i}^{\prime}(\psi)\right)(\nabla \psi)^{2} C_{i} \\
& +D_{i}(\psi) Q_{i}(\psi) \Delta \psi C_{i}+D_{i}(\psi) Q_{i}(\psi) \nabla \psi \cdot \nabla C_{i}
\end{aligned}
$$

We use (3.1) and get the estimate

$$
\begin{aligned}
& \left\|D_{i}(\psi) Q_{i}(\psi) \nabla \psi \cdot \nabla C_{i}\right\|_{L^{p}\left(0, T ; L^{p}(\Omega)\right)} \\
& \quad \leq T^{\frac{1}{p}} \Lambda\|\nabla \psi\|_{C\left([0, T] ; L^{p}(\Omega)\right)}\left\|\nabla C_{i}\right\|_{C([0, T] ; C(\bar{\Omega}))} \\
& \quad \leq T^{\frac{1}{p}} \Lambda\|\nabla \psi\|_{C\left([0, T] ; L^{p}(\Omega)\right)}\left\|C_{i}\right\|_{W_{p}^{2,1}\left(Q_{T}\right)}
\end{aligned}
$$

where the last inequality is true for $n+2<p<\infty$ (see [8] for an explanation of our special choice of $p$ ). The other terms in (6.18) and the last $L^{p}$-norm in (6.17) may be estimated similarily. Again we can say that there exists a constant $\Lambda_{2}>0$ just depending on known quantities such that

$$
\begin{gathered}
\left\|\operatorname{div}\left\{D_{i}(\psi) Q_{i}(\psi) \nabla \psi C_{i}\right\}\right\|_{L^{p}\left(0, T ; L^{p}(\Omega)\right)}+\left\|D^{\prime}(\psi) \nabla \psi \cdot \nabla C_{i}\right\|_{L^{p}\left(0, T ; L^{p}(\Omega)\right)} \\
\leq T^{\frac{1}{p}} \Lambda_{2}\left\|C_{i}\right\|_{W_{p}^{2,1}\left(Q_{T}\right)}
\end{gathered}
$$

Thus, in summary,

$$
\left(1-T^{\frac{1}{p}} \frac{K_{0} \Lambda_{2}}{2}\right)\left\|C_{i}\right\|_{W_{p}^{2,1}\left(Q_{T}\right)} \leq \frac{K_{0}}{2}\left(\frac{\Lambda_{0}}{2}+T^{\frac{1}{p}} \Lambda_{1}\right) .
$$

We choose $T_{f} \in\left(0, T_{1}\right]$ such that $0<T_{f}^{\frac{1}{p}} \leq \min \left\{\frac{1}{K_{0} \Lambda_{2}}, \frac{\Lambda_{0}}{2 \Lambda_{1}}\right\}$. Then (cf. (6.1)) we get $\left\|C_{i}\right\|_{W_{p}^{2,1}\left(Q_{T_{f}}\right)} \leq k_{1}$ for $i \in J$ and so $Z: X_{T_{f}} \rightarrow X_{T_{f}}$.

Step III: We we proceed with

Lemma 5. The mapping $Z: X_{T_{f}} \rightarrow X_{T_{f}}$ is compact and continuous.

Proof. At first we note that the embedding

$$
W_{p}^{1}\left(0, T_{f} ; W_{p}^{2}(\Omega)\right) \subset C\left(\left[0, T_{f}\right] ; W_{p}^{1}(\Omega)\right)
$$

is compact. Thus, from (6.8) the mapping is compact in the second variable. Now let $\left\{\left(\mathcal{C}_{i}^{m}\right)_{i \in J}, \phi_{m}\right\}_{m \in \mathbb{N}} \subset X_{T_{f}}$.

1. From the compact embedding of $W_{p}^{2,1}\left(Q_{T_{f}}\right)$ into the space $L^{p}\left(0, T_{f} ; W_{p}^{1}(\Omega)\right)$ there follows the existence of a subsequence $\mathcal{C}_{i}^{n} \rightarrow \mathcal{C}_{i}$ in $L^{p}\left(0, T_{f} ; W_{p}^{1}(\Omega)\right)$ for $n \rightarrow \infty$. This is also true for the cuts, i.e.

$$
\mathcal{C}_{i}^{n+} \rightarrow \mathcal{C}_{i}^{+} \quad \text { in } L^{p}\left(0, T_{f} ; W_{p}^{1}(\Omega)\right) \text { for } n \rightarrow \infty
$$


2. If we apply Lemma 2 to the equation in (6.5), there follows the existence of a subsequence

$$
C_{A}^{n} \rightarrow C_{A} \quad \text { in } C\left(\left[0, T_{f}\right] ; C(\bar{\Omega})\right) \text { for } n \rightarrow \infty
$$

3. From (6.19) - (6.21) there follows the convergence of a subsequence

$$
\psi_{n} \rightarrow \psi \quad \text { in } C\left(\left[0, T_{f}\right] ; W_{p}^{1}(\Omega)\right) \text { for } n \rightarrow \infty
$$

Let $C_{i}^{n}, C_{i} \quad(i \in J)$ be the solutions of problem (6.7). We set

$$
\begin{aligned}
\overline{\mathcal{C}}_{i}^{n} & =\mathcal{C}_{i}^{n+}-\mathcal{C}_{i}^{+} \quad(i \in J) \\
\bar{C}_{i}^{n} & =C_{i}^{n}-C_{i} \quad\left(i \in J \cup J^{\prime}\right) \\
\bar{\psi}^{n} & =\psi_{n}-\psi
\end{aligned}
$$

In $Q_{T_{f}}$ we consider the system for $\bar{C}_{i}^{n} \quad(i \in J)$ which is

$$
\frac{\partial \bar{C}_{i}^{n}}{\partial t}-\operatorname{div}\left\{B_{1} \nabla \bar{C}_{i}^{n}\right\}+B_{2} \nabla \bar{C}_{i}^{n}+B_{3} \bar{C}_{i}^{n}=\bar{F}_{i}^{n}
$$

where

$$
\begin{aligned}
\bar{F}_{i}^{n}= & A_{1}\left(D_{i}\left(\psi_{n}\right)-D_{i}(\psi)\right)+A_{2}\left(D_{i}^{\prime}\left(\psi_{n}\right)-D_{i}^{\prime}(\psi)\right) \\
& +A_{3}\left(Q_{i}\left(\psi_{n}\right)-Q_{i}(\psi)\right)+A_{4}\left(Q_{i}^{\prime}\left(\psi_{n}\right)-Q_{i}^{\prime}(\psi)\right) \\
& +A_{5} \nabla \bar{\psi}^{n}+A_{6} \Delta \bar{\psi}^{n} \\
& +R_{i}\left(\left(\mathcal{C}_{k}^{n+}\right)_{k \in I}, C_{A}^{n}, \psi_{n}\right)-R_{i}\left(\left(\mathcal{C}_{k}^{+}\right)_{k \in I}, C_{A}, \psi\right)
\end{aligned}
$$

and with the boundary conditions $\nabla \bar{C}_{i}^{n} \cdot \mathbf{n}=0$ on $\Sigma_{T}$ and zero intial conditions. We do not discuss the coefficients $A$ and $B$ (supplied with indices) appearing in the linear equations in detail, but mention that they belong at least to the space $C\left(\left[0, T_{f}\right] ; L^{p}(\Omega)\right)$, which we will see in a minute. The parabolic theory (see [8]) yields

$$
\sum_{i \in J}\left\|\bar{C}_{i}^{n}\right\|_{W_{p}^{2,1}\left(Q_{T_{f}}\right)} \leq \Lambda \sum_{i \in J}\left\|\bar{F}_{i}^{n}\right\|_{L^{p}\left(0, T_{f} ; L^{p}(\Omega)\right)} .
$$

In order to show convergence of the left-hand side we have to estimate the right-hand side of (6.25) with the help of (5.1) as well as (6.20) and (6.21). For this we use the mean value theorem to get

$$
Q_{i}\left(\psi_{n}\right)-Q_{i}(\psi)=\int_{0}^{1} Q_{i}^{\prime}(\tilde{\psi}) d s \bar{\psi}^{n}
$$


(the same with the remaining coefficients which depent on $\psi$ ) where $\tilde{\psi}=$ $s \psi_{n}+(1-s) \psi$. Representative, we estimate the term $A_{3}\left(Q_{i}\left(\psi_{n}\right)-Q_{i}(\psi)\right)$ where a short calculation gives

$$
A_{3}=D_{i}^{\prime}(\psi) C_{i}(\nabla \psi)^{2}+D_{i}(\psi) \nabla C_{i} \cdot \nabla \psi+D_{i}(\psi) C_{i} \Delta \psi
$$

Therein, $\Delta \psi \in C\left(\left[0, T_{f}\right] ; L^{p}(\Omega)\right)$ whereas due to embedding results the otherfunctions are continuous and together we get $A_{3} \in C\left(\left[0, T_{f}\right] ; L^{p}(\Omega)\right)$. Thus,

$$
\begin{aligned}
& \left\|A_{3} \int_{0}^{1} Q_{i}^{\prime}(\tilde{\psi}) d s \bar{\psi}^{n}\right\|_{L^{p}\left(0, T_{f} ; L^{p}(\Omega)\right)} \\
& \quad \leq \Lambda\left(\left\|\bar{\psi}^{n}\right\|_{L^{p}\left(0, T_{f} ; L^{p}(\Omega)\right)}+\left\|\Delta \psi \bar{\psi}^{n}\right\|_{L^{p}\left(0, T_{f} ; L^{p}(\Omega)\right)}\right) \\
& \quad \leq \Lambda\left(1+\|\Delta \psi\|_{C\left(\left[0, T_{f}\right] ; L^{p}(\Omega)\right)}\right)\left\|\bar{\psi}^{n}\right\|_{L^{p}\left(0, T_{f} ; C(\bar{\Omega})\right)} \\
& \quad \leq \Lambda\left\|\bar{\psi}^{n}\right\|_{L^{p}\left(0, T_{f} ; W_{p}^{2}(\Omega)\right)} \\
& \quad \leq \Lambda\left\|_{i} C_{i}^{n}\right\|_{L^{p}\left(0, T_{f} ; L^{p}(\Omega)\right)}+\left\|\bar{C}_{A}^{n}\right\|_{L^{p}\left(0, T_{f} ; L^{p}(\Omega)\right)} .
\end{aligned}
$$

The other terms in (6.25) may be estimated similarily. Thus, we are able to show that there exists a constant $\Lambda>0$ satisfying

$$
\begin{aligned}
& \sum_{i \in J}\left\|\bar{F}_{i}^{n}\right\|_{L^{p}\left(0, T_{f} ; L^{p}(\Omega)\right)} \\
& \quad \leq \Lambda\left(\sum_{i \in J}\left\|_{i} C_{i}^{n}\right\|_{L^{p}\left(0, T_{f} ; L^{p}(\Omega)\right)}+\left\|\bar{C}_{A}^{n}\right\|_{L^{p}\left(0, T_{f} ; L^{p}(\Omega)\right)}\right)
\end{aligned}
$$

which implies convergence in the left-hand side of (6.25). This and (6.19) prove the compactness of the mapping $Z$.

The continuity of the Mapping $Z$ can be obtained by similar arguments. More precisely, we take a sequence $\left\{\left(\mathcal{C}_{i}^{n}\right)_{i \in J}, \phi_{n}\right\}_{n \in \mathbb{N}} \subset X_{T_{f}}$ and from this we get for the cuts $\mathcal{C}_{i}^{n+}$ that

$$
\mathcal{C}_{i}^{n+} \rightarrow \mathcal{C}_{i}^{+} \quad \text { in } L^{p}\left(0, T_{f} ; W_{p}^{1}(\Omega)\right) \cap W_{p}^{1}\left(0, T_{f} ; L^{p}(\Omega)\right)
$$

as well as

$$
\phi_{n} \rightarrow \phi \quad \text { in } C\left(\left[0, T_{f}\right] ; W_{p}^{1}(\Omega)\right)
$$

for $n \rightarrow \infty$. We consider the differences in equations $(6.5)-(6.7)$, use inequalities (5.1) and (6.12) to get the continuity of the mapping $Z$

From steps I - III we conclude the existence of a local, non-negative solution of Problem $\left(\mathrm{P}^{+}\right)$. 
6.3 Uniqueness of problem (P). The solution of problem $\left(\mathrm{P}^{+}\right)$obviously solves problem $(\mathrm{P})$, too. In order to show that the (non-negative) solution, which we denote by $C_{i}^{1}, \psi^{1} \quad\left(i \in J \cup J^{\prime}\right)$, is the only one, we assume the existence of another, not necessarily non-negative solution $C_{i}^{2}, \psi^{2} \quad(i \in J \cup$ $\left.J^{\prime}\right)$. We again consider the system for the respective differences $\bar{C}_{i}=C_{i}^{1}-$ $C_{i}^{2} \quad\left(i \in J \cup J^{\prime}\right)$ which is for $i \in J$ exactly the same as (6.24) if we replace the cuts $\left(\mathcal{C}_{k}^{n+}\right)_{k \in J}$ and $\left(\mathcal{C}_{k}^{+}\right)_{k \in J}$ in the right-hand side $\bar{F}_{i}$ by the solution vectors $\left(C_{k}^{1}\right)_{k \in J}$ and $\left(C_{k}^{2}\right)_{k \in J}$, respectively. We test the $i$-th equation with $\bar{C}_{i}$, and if we set

$$
\|\cdot\|_{V^{1,0}\left(Q_{t}\right)}^{2}=\sup _{0 \leq \tau \leq t}\|\cdot\|_{L^{2}(\Omega)}^{2}+\|\cdot\|_{L^{2}\left(0, t ; H^{1}(\Omega)\right)}^{2}
$$

we get with the same methods presented in the previous sections the inequality

$$
\begin{aligned}
\left\|\bar{C}_{i}\right\|_{V^{1,0}\left(Q_{t}\right)}^{2} \leq & \Lambda\left(\Lambda_{\varepsilon} \sum_{k \in J \cup J^{\prime}} \int_{0}^{t}\|g(s)\|_{L^{\infty}(\Omega)}^{2}\left\|\bar{C}_{k}(s)\right\|_{L^{2}(\Omega)}^{2} d s\right. \\
& \left.+\frac{\varepsilon}{2} \sum_{k \in J}\left\|\bar{C}_{k}\right\|_{V^{1,0}\left(Q_{t}\right)}^{2}+\|\bar{\psi}\|_{L^{2}\left(0, t ; H^{1}(\Omega)\right)}\right)
\end{aligned}
$$

with some $g \in L^{2}\left(0, t ; L^{\infty}(\Omega)\right)$ and $\varepsilon>0$ arbitrarily. Similarily, we deduce

$$
\left\|\bar{C}_{A}(t)\right\|_{L^{2}(\Omega)}^{2} \leq \Lambda\left(\sum_{k \in J \cup J^{\prime}} \int_{0}^{t}\|g(s)\|_{L^{\infty}(\Omega)}^{2}\left\|\bar{C}_{k}(s)\right\|_{L^{2}(\Omega)}^{2} d s+\|\bar{\psi}\|_{L^{2}\left(0, t ; H^{1}(\Omega)\right)}\right) .
$$

Summation over $i \in J \cup J^{\prime}$, the choice of a suitable $\varepsilon>0$ and the application of the stability estimate (5.1), which remains valid if only one of the concentrations is non-negative, yield

$$
\sum_{i \in J \cup J^{\prime}}\left\|\bar{C}_{i}(t)\right\|_{L^{2}(\Omega)}^{2} \leq \Lambda \sum_{i \in J \cup J^{\prime}} \int_{0}^{t}\left(1+\|g\|_{L^{\infty}(\Omega)}^{2}\right)\left\|\bar{C}_{i}\right\|_{L^{2}(\Omega)}^{2} d s
$$

for all $t \in\left[0, T_{f}\right]$. So, we conclude with Gronwall's lemma $\sum_{i \in J \cup J^{\prime}}\left\|\bar{C}_{i}(t)\right\|_{L^{2}(\Omega)}^{2}=$ 0 and this in turn yields with (5.1) that $\left\|\psi_{1}(t)-\psi_{2}(t)\right\|_{H^{1}(\Omega)}=0$. This proves the unique solvability of problem $(\mathrm{P})$ which completes the proof of Theorem 


\section{References}

[1] Fahey, P. M., Griffin, P. B. and J. D. Plummer: Point defects and dopant diffusion in silicon. Rev. Mod. Phys. 61 (1989), 290 - 383.

[2] Ghaderi, K. and G. Hobler: Simulation of phosphorus diffusion in silicon using a pair diffusion model with a reduced number of parameters. J. Electrochem. Soc. 142 (1995), $1654-1658$.

[3] Gilbarg, D. and N. S. Trudinger: Elliptic Partial Differential Equations of Second Order. Berlin et al.: Springer-Verlag 1977.

[4] Glitzky, A. and R. Hünlich: Global properties of pair diffusion models. Preprint. Berlin: WIAS preprint No 587 (2000).

[5] Grisvard, P.: Elliptic Problems in Nonsmooth Domains. Boston-London-Melbourne: Pitman 1985.

[6] Höfler, A. and N. Strecker: On the coupled diffusion of dopants and silicon point defects. Report. Zürich: Swiss Federal Inst. Techn., Integrated Sys. Lab., Technical Report 94/11 (1994).

[7] Hünlich, R. and A. Glitzky: On energy estimates for electro-reaction-diffusion equations arising in semiconductor technology. In: Partial differential equations, Theory and numerical solution (eds.: W. Jäger et al.; Chapman Hall/CRC Res. Notes Math.: Vol. 406). Boca Raton: Chapman 2000, pp. 158 - 174.

[8] Ladyženskaja, O. A., Solonnikov, V. A. and N. N. Ural'ceva: Linear and Quasilinear Equations of Parabolic Type. Providence (R.I., USA): Amer. Math. Soc. 1968.

[9] Ladyženskaja, O. A. and N. N. Ural'ceva: Linear and Quasilinear Equations of Elliptic Type. New York - London: Acad. Press 1968.

[10] Lions, J. L. and E. Magenes: Non-Homogeneous Boundary Value Problems and Applications, Vol. I and II. Berlin et al.: Springer-Verlag 1973.

[11] Martin, R. H.: Nonlinear Operators and Differential Equations in Banach Spaces. New York et al.: J. Wiley 1976.

[12] Merz, W.: Analysis und Numerische Berechnung der Diffusion von Fremdatomen in Homogenen Strukturen. Habilitationsschrift. München (Germany): Techn. Univ. 1998.

[13] Merz, W.: Strong solutions for reaction-drift-diffusion problems in semiconductor devices. ZAMM (to appear).

[14] Merz, W. and A. Glitzky: Single dopant diffusion in semiconductor technology. Preprint. München: Techn. Univ. and Augsburg: Univ., Preprint SFB-438, Nr. 0011 (2000), pp. $1-23$.

[15] Merz, W., Glitzky, A., Hünlich, R. and K. Pulverer: Strong solutions for pair diffusion models in homogeneous semiconductors equations in homogeneous structures. Preprint. München: Tech. Univ. and Augsburg: Univ., Preprint SFB-438-9921 (1999), pp. 1 - 32.

[16] Trudinger, N. S.: On imbeddings into Orlicz spaces and some applications. J. Math. Mech. 17 (1967), $473-483$. 
114 R. Bader and W. Merz

Received 08.11.2000; in revised form 04.10.2001 